ISSN 0103-8478

\title{
Farmacocinética do propofol em nanoemulsão em cães
}

\author{
Pharmacokinetics of propofol nanoemulsion in dogs
}

\author{
Rafael Messias Luiz ${ }^{\mathrm{I}}$ Martielo Ivan Gehrcke ${ }^{\mathrm{II}}$ Marcos Paulo Antunes de Lima ${ }^{\mathrm{II}}$ Anicleto Poli ${ }^{\mathrm{III}}$ \\ Aury Nunes de Moraes ${ }^{\mathrm{II}}$ Felipe Hertzing Farias ${ }^{\mathrm{II}}$ Nilson Oleskovicz $^{\mathrm{I}^{*}}$
}

\section{RESUMO}

O propofol em nanoemulsão é uma nova formulação constituída por sistemas nanoemulsionados, caracterizado pela ausência do veículo lipídico. A modificação do veículo pode acarretar alterações farmacocinéticas, resultando em diferentes taxas de distribuição e excreção do propofol. O objetivo deste estudo foi avaliar a farmacocinética de uma nova formulação de propofol em nanoemulsão do tipo óleo em água, comparando com a formulação tradicional em emulsão lipídica. Foram utilizadas seis cadelas sem raça definida, castradas $(10,7 \pm 1,5 \mathrm{~kg})$, em estudo aleatório e de autocontrole, que receberam as duas formulações de propofol com intervalo de 30 dias entre os tratamentos, sendo administrada uma dose bolus de $8 \mathrm{mg} \mathrm{kg}^{-1}$, seguida de infusão contínua por 60 minutos na taxa de $0,4 \mathrm{mg} \mathrm{kg}^{-1} \mathrm{~min}^{-1}$. Amostras de sangue arterial foram colhidas momentos antes da indução (0), $2,5,10,15,30$ e 60 minutos após a dose bolus e após o término da infusão nos tempos 5, 10,15,30,60 e 90 minutos e 2, 3, 4, 6, 10 e 24 horas. Não foram encontradas diferenças significativas entre os parâmetros farmacocinéticos de volume de distribuição, clearance, constante de eliminação, meia-vida e constantes de distribuição, demonstrando que o propofol em nanoemulsão não apresenta alteração farmacocinética em relação à formulação tradicional.

Palavras-chave: propofol, farmacocinética, cães, nanoemulsão.

\section{ABSTRACT}

Propofol nanoemulsion is a new formulation consisting of nanoemulsified systems, characterized by the absence of lipid vehicle. Changes in drug vehicle may alter the pharmacokinetics and result in different distribution and elimination rates of propofol. The aim of this study was to evaluate the pharmacokinetics of a new oil in water propofol nanoemulsion formulation, compared to the traditional lipid emulsion. Six neutered female mixed-breed dogs were used $(10.7 \pm 1.5 \mathrm{~kg})$ in a randomized and self control trial with 30 days interval. They received both propofol formulations administered as a bolus dose of $8 \mathrm{mg} \mathrm{kg}^{-1}$ followed by 60 minutes continuous infusion at $0.4 \mathrm{mg}$ $\mathrm{kg}^{-1} \mathrm{~min}^{-1}$ rate. Arterial blood samples were collected just before induction (0), 2, 5, 10, 15, 30, and 60 minutes after the bolus dose, and after the end of the infusion at five, 10, 15, 30,60, and 90 minutes and 2, 3, 4, 6, 10, and 24 hours. There were no significant differences in the evaluated pharmacokinetics parameters that included volume of distribution, clearance, elimination rate constant, half-life, and distribution constants, demonstrating that propofol nanoemulsion has no pharmacokinetics differences when compared to the traditional formulation.

Key words: propofol, pharmacokinetics, dogs, nanoemulsion.

\section{INTRODUÇÃO}

O propofol é um anestésico intravenoso de curta duração, introduzido na rotina clínica como agente de indução anestésica alternativo aos agentes barbitúricos no final da década de 1980 (WHITE, 2008). As características farmacocinéticas de rápido início de ação, curta duração, rápido metabolismo extra-hepático e clearance tornam o propofol um agente de eleição para indução e manutenção anestésica (TRAPANI et al., 2004), sendo uma alternativa à anestesia inalatória, podendo ser utilizada por bolus intermitente, infusão contínua (IC) ou infusão alvo-controlada (BETHS et al., 2001).

A atual formulação de propofol foi desenvolvida na década de 1980, como uma emulsão óleo em água, composta por óleo de soja (10\%),

ISetor Palotina, Hospital Veterinário, Universidade Federal do Paraná (UFPR), Palotina, PR, Brasil.

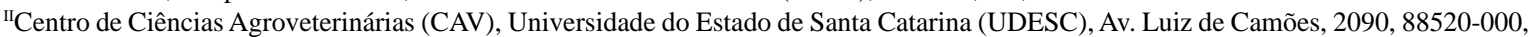
Lages, SC, Brasil. E-mail: a2no@ cav.udesc.br.*Autor para correspondência.

IIIDepartamento de Farmacologia, Campus Universitário Trindade, Universidade Federal de Santa Catarina (UFSC), Florianópolis, SC, Brasil. Recebido 07.05.12 Aprovado 16.03.13 Devolvido pelo autor 06.06.13 CR-2012-0151.R1 
glicerol $(2,25 \%)$, fosfato de ovo purificado $(1,2 \%)$ e EDTA $(0,005 \%)$ (KOTANI et al., 2008). Em emulsão lipídica, o propofol apresenta-se como uma solução de baixa estabilidade física, com curto tempo de estocagem, sendo um potencial meio de cultura microbiológico, necessitando ser armazenado sob temperatura controlada (TRAPANI et al., 2004).

As nanoemulsões apresentam-se como formulação alternativa para princípios ativos lipofílicos e pouco solúveis como o propofol (DATE \& NAGARSENKER, 2008). A nanoemulsão é caracterizada por ser transparente, incolor e termodinamicamente estável, com partículas medindo entre 10 e 50ๆm (MOREY et al., 2006b). Os veículos nanoemulsionados são estéreis, apirogênicos, biocompatíveis, não irritantes e não hemolíticos (DATE \& NAGARSENKER, 2008). Em geral, por serem altamente hidrossolúveis, as nanoemulsões apresentam mínima distribuição tecidual (KIM et al., 2007), fato que pode acarretar em alteração na farmacocinética do fármaco.

Diante do exposto, o presente estudo pretende estabelecer os parâmetros farmacocinéticos do propofol em nanoemulsão para a espécie canina e verificar as possíveis diferenças farmacocinéticas entre esta e a formulação em emulsão lipídica.

\section{MATERIAL E MÉTODOS}

Foram utilizadas seis cadelas hígidas, sem raça definida (SRD), castradas, com idade entre 2 e 4 anos e peso médio de $10,7 \pm 1,5 \mathrm{~kg}$, de forma randomizada, sendo as unidades experimentais auto controle, com intervalo mínimo de 30 dias entre as fases de experimentação.

Após período de adaptação de seis meses, os cães foram submetidos a jejum alimentar de 12 horas e hídrico de duas horas, foram instrumentados após indução anestésica com isofluorano a $5 \mathrm{~V} \%$, seguida por intubação e mantidos em sistema com reinalação parcial de gases com 1 a $1,5 \mathrm{~V} \%$ de isofluorano diluído em oxigênio a $100 \%$, na taxa de $50 \mathrm{~mL} \mathrm{~kg}^{-1} \mathrm{~min}^{-1}$ em regime de ventilação espontânea.

Na sequência, realizou-se bloqueio anestésico local na região da artéria metatarsiana dorsal com $0,3 \mathrm{~mL}$ de lidocaína $2 \%$ sem vasoconstritor, seguida da cateterização com cateter $22 \mathrm{G}$, para a colheita de sangue para as análises cromatográficas. Da mesma forma, foram cateterizadas as duas veias cefálicas com cateter $22 \mathrm{G}$, para administração de solução de cloreto de sódio a $0,9 \%$ em bomba de infusão peristáltica e administração de propofol através de bomba de infusão de seringa.
Uma hora após a recuperação anestésica, iniciou-se o período experimental. Foi instituído fluidoterapia com solução de cloreto de sódio a $0,9 \%$ na taxa de $5 \mathrm{~mL} \mathrm{~kg}^{-1} \mathrm{~h}^{-1}$ e iniciada a administração de propofol a 1\% em emulsão lipídica (EMU) (Propovan ${ }^{\circledR}$, Cristália Produtos Químicos Farmacêuticos Ltda, $10 \mathrm{mg} \mathrm{mL}^{-1}$ ) ou em nanoemulsão (NANO) (Propovet ${ }^{\circledR}$, Ouro Fino Saude Animal Ltda, Lote Piloto 001/2009, $10 \mathrm{mg} \mathrm{mL}^{-1}$ ), na dose estipulada por CORRÊA (2010) de $8 \mathrm{mg} \mathrm{kg}^{-1}$ em bolus para indução, administrado no período de um minuto, seguido de manutenção por infusão contínua na taxa de $0,4 \mathrm{mg} \mathrm{kg}^{-1} \mathrm{~min}^{-1}$ durante uma hora, sendo empregada a mesma formulação para indução e manutenção.

Após o período experimental, os cães permaneceram confinados, recebendo água ad libitum após duas horas e alimentação controlada após cada colheita, a partir de quatro horas do término da infusão contínua de propofol. Para a avaliação cinética do propofol, foram colhidos $3,0 \mathrm{~mL}$ de sangue arterial em tubos com EDTA. As amostras foram colhidas em duas etapas: durante a IC de propofol (momento antes da indução (0), 2, 5, 10, 15, 30 e 60 minutos após a dose bolus) e após o término da IC $(5,10,15$, 30, 60 e 90 minutos e 2, 3, 4, 6, 10 e 24 horas). As amostras foram centrifugadas a 3000rpm durante 10 minutos, sendo o plasma armazenado em tubos do tipo eppendorf em freezer a $-80^{\circ} \mathrm{C}$ até a realização das análises cromatográficas por cromatografia líquida de alta eficiência (CLAE), com detecção por Espectrometria de Massas (MS/MS), segundo técnica desenvolvida e validada pelo laboratório BIOTEC Biotecnologia, Campinas-SP.

As curvas de concentração plasmática (Cp) de propofol em função do tempo foram obtidas para cada grupo/animal, para as duas formulações, sendo confeccionados gráficos $\mathrm{Cp}$ versus tempo para os períodos de IC e após o término da IC de propofol através do programa "Graphpad Prism $5^{\circledR}$ ". A concentração plasmática máxima $\left(\mathrm{C}_{\max }\right)$ foi obtida a partir de cada curva no período de IC em ambos os grupos. A área sob a curva individual de concentração do fármaco versus tempo de 0 a $24 \mathrm{~h}\left(\mathrm{ASC}_{0-24}\right)$ foi calculada usando-se o método trapezoidal. A extrapolação da $\mathrm{ASC}_{0-24}$ ao infinito $\left(\mathrm{ASC}_{0-\infty}\right)$ foi obtida pela adição do valor $\mathrm{C}_{24} / \mathrm{ke}$ à $\mathrm{ASC}_{0-24}$ calculada (em que $\mathrm{C}_{24}$ corresponde à concentração plasmática 24 horas após a administração do propofol e $\mathrm{k}_{\mathrm{e}}$ corresponde à constante de eliminação).

As variáveis farmacocinéticas de constante de eliminação compartimental $(\alpha, \beta$ e $\gamma)$, meia-vida de distribuição, eliminação plasmática e terminal $\left(\mathrm{T}_{1 / 2 \alpha}, \mathrm{T}_{1 / 2 \beta}\right.$ e $\left.\mathrm{T}_{1 / 2 \gamma}\right)$ e interceptos (A, B e C) e tempo de 
residência médio (TRM) foram calculadas por meio do programa Pharmkit para Windows. Os valores de clearance metabólico, do segundo e terceiro compartimento e clearance total $\left(\mathrm{Cl}_{1}, \mathrm{Cl}_{2}, \mathrm{Cl}_{3}\right.$ e $\left.\mathrm{Cl}\right)$, volume de distribuição central, do segundo e terceiro compartimento e no estado de equilíbrio $\left(\mathrm{V}_{1}, \mathrm{~V}_{2}\right.$, $\mathrm{V}_{3}$ e $\left.\mathrm{V}_{\mathrm{dss}}\right)$ constantes de distribuição $\left(\mathrm{K}_{12}\right.$ e $\left.\mathrm{K}_{13}\right)$, de redistribuição $\left(K_{21}\right.$ e $\left.K_{31}\right)$ e eliminação $\left(K_{10}\right)$, foram calculados por meio da "Planilha Convert" para Excel.

A análise estatística foi realizada por meio do programa "SigmaStat 3.0" sendo aplicada ANOVA de uma via com repetições múltiplas, seguida de teste de Bonferroni para comparação das concentrações plasmáticas do mesmo grupo. Para avaliação das concentrações plasmáticas e dos parâmetros farmacocinéticos entre EMU e NANO, foi empregado teste T pareado com nível de significância de 5\%.

\section{RESULTADOS E DISCUSSÃO}

As curvas farmacocinéticas obtidas para as formulações foram semelhantes (Figura 1A e 1B), sendo observado comportamento cinético tricompartimental. As Cp médias observadas durante o período de infusão (Figura 1A) são justificadas pelas características farmacotécnicas das formulações em que a nanoemulsão com um tamanho reduzido de

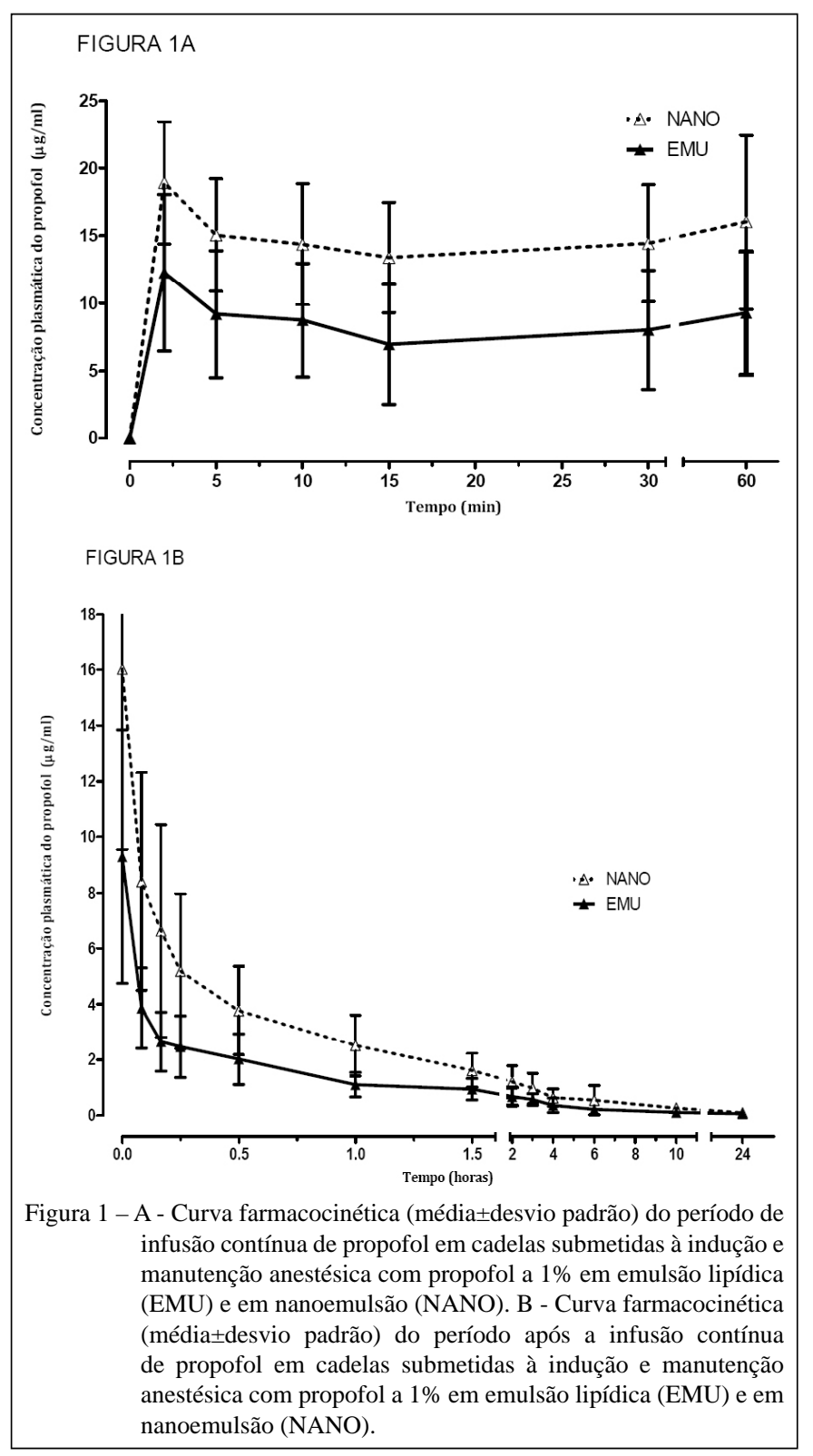

Ciência Rural, v.43, n.8, ago, 2013. 
suas partículas (KIM et al., 2007) e menor afinidade lipídica (JUNG et al., 2010) apresenta menor volume de distribuição, gerando maiores concentrações, quando comparada à emulsão lipídica, sugerindo que a nanoemulsão apresenta maior potência anestésica, quando administrada nas mesmas doses, como relatado por GEHRCKE (2011).

Não foram observadas diferenças significativas nos parâmetros farmacocinéticos entre as formulações (Tabela 1). DATE \& NAGARSENKER (2008) relatam que o surfactante não altera a farmacodinâmica em ratos, permanecendo o propofol nanoemulsionado com características cinéticas semelhantes à emulsão lipídica. A ausência de diferença entre os parâmetros é relatada em outras formulações nanoemulsionadas de propofol como a microemulsão de MOREY et al. (2006b), a formulação micelar testada por RAVENELLE et al. (2008), a formulação em pró-droga avaliada por CLEALE et al. (2009) e a microemulsão de LEE et al. (2009).

Os resultados obtidos demonstram que a farmacocinética não sofreu alteração em detrimento da substituição do veículo lipídico pelo Solutol HS 15 , entretanto, a escolha de animais sem raça definida (SRD) pode ter sido a fonte do grande desvio padrão observado. Segundo ZORAN et al. (1993), a raça altera a farmacocinética, sendo possível observar variações em alguns parâmetros descritos por LEE et al. (2009) para cães de raça.

Tabela 1 - Parâmetros farmacocinéticos (média \pm desvio padrão) de cadelas submetidas à indução e manutenção anestésica com propofol a $1 \%$ em emulsão lipídica (EMU) e em nanoemulsão (NANO).

\begin{tabular}{|c|c|c|c|c|}
\hline & Média & DP & Média & DP \\
\hline $\mathrm{Cmax}\left(\mu \mathrm{g} \mathrm{ml}^{-1}\right)$ & 13,03 & 5,79 & 20,02 & 4,71 \\
\hline $\mathrm{ASC}_{0-24}\left(\mathrm{ng} \mathrm{min} \mathrm{min}^{-1} \mathrm{ml}^{-1}\right)$ & 971,96 & 337,50 & 1703,66 & 613,36 \\
\hline $\mathrm{ASC}_{0-\infty}\left(\mathrm{ng} \min ^{-1} \mathrm{ml}^{-1}\right)$ & 972,90 & 337,48 & 1704,88 & 613,98 \\
\hline $\operatorname{MRT}(\mathrm{h})$ & 9,09 & 2,94 & 6,93 & 1,51 \\
\hline $\mathrm{V}_{1}\left(\mathrm{~L} \mathrm{~kg}^{-1}\right)$ & 3,75 & 2,11 & 2,38 & 1,16 \\
\hline $\mathrm{V}_{2}\left(\mathrm{~L} \mathrm{~kg}^{-1}\right)$ & 9,07 & 6,52 & 4,02 & 3,42 \\
\hline $\mathrm{V}_{3}\left(\mathrm{~L} \mathrm{~kg}^{-1}\right)$ & 20,61 & 10,12 & 15,00 & 9,15 \\
\hline $\mathrm{V}_{\mathrm{dss}}\left(\mathrm{L} \mathrm{kg}^{-1}\right)$ & 44,66 & 25,96 & 21,40 & 12,99 \\
\hline $\mathrm{Cl}_{1}\left(\mathrm{ml} \mathrm{kg}^{-1} \min ^{-1}\right)$ & 81,95 & 26,98 & 48,86 & 29,50 \\
\hline $\mathrm{Cl}_{2}\left(\mathrm{ml} \mathrm{kg}^{-1} \min ^{-1}\right)$ & 572,45 & 169,95 & 338,15 & 382,01 \\
\hline $\mathrm{Cl}_{3}\left(\mathrm{ml} \mathrm{kg}{ }^{-1} \min ^{-1}\right)$ & 73,04 & 37,0 & 38,81 & 23,23 \\
\hline $\mathrm{Cl}\left(\mathrm{ml} \mathrm{kg}{ }^{-1} \min ^{-1}\right)$ & 791,23 & 231,67 & 512,04 & 357,02 \\
\hline $\mathrm{T}_{1 / 2 \alpha}(\min )$ & 2,06 & 1,34 & 4,03 & 2,27 \\
\hline $\mathrm{T}_{1 / 2 \beta}(\min )$ & 58,14 & 31,07 & 52,35 & 17,68 \\
\hline $\mathrm{T}_{1 / 2 \gamma}(\min )$ & 660,94 & 304,67 & 551,79 & 113,13 \\
\hline $\mathrm{K}_{10}(\min )$ & 0,0253 & 0,0103 & 0,0207 & 0,0082 \\
\hline $\mathrm{K}_{12}(\min )$ & 0,2811 & 0,1576 & 0,1241 & 0,0836 \\
\hline $\mathrm{K}_{13}(\min )$ & 0,0221 & 0,0127 & 0,0452 & 0,0692 \\
\hline $\mathrm{K}_{21}(\min )$ & 0,1234 & 0,0610 & 0,0772 & 0,0342 \\
\hline $\mathrm{K}_{31}(\min )$ & 0,0033 & 0,0035 & 0,0028 & 0,0011 \\
\hline A & 7,84 & 3,32 & 10,75 & 4,16 \\
\hline B & 2,35 & 1,21 & 4,41 & 2,27 \\
\hline $\mathrm{C}$ & 0,35 & 0,38 & 0,62 & 0,41 \\
\hline$\alpha\left(h^{-1}\right)$ & 26,32 & 12,98 & 13,52 & 7,17 \\
\hline$\beta\left(\mathrm{h}^{-1}\right)$ & 0,91 & 0,50 & 0,87 & 0,26 \\
\hline$\gamma\left(\mathrm{h}^{-1}\right)$ & 0,08 & 0,06 & 0,08 & 0,01 \\
\hline
\end{tabular}

$\mathrm{C}_{\max }$ : concentração plasmática máxima; $\mathrm{AUC}_{0-24}$ : área sob a curva do tempo 0 até 24 horas; $\mathrm{ASC}_{0-\infty}$ área sob a curva do tempo 0 ao infinito; MRT: tempo de residência médio; $\mathrm{V}_{1}$ : volume de distribuição central; $\mathrm{V}_{2}$ : volume de distribuição do segundo compartimento; $\mathrm{V}_{3}$ : volume de distribuição do terceiro compartimento; $\mathrm{V}_{\mathrm{dss}}$ : volume de distribuição no estado de equilíbrio; $\mathrm{Cl}_{1}$ : clearance metabólico; $\mathrm{Cl}_{2}$ : clearance do segundo compartimento; $\mathrm{Cl}_{3}$ : clearance do terceiro compartimento; $\mathrm{Cl}$ : clearance total; $\mathrm{T}_{1 / 2 \alpha}$ : meia vida de distribuição; $\mathrm{T}_{1 / 2 \beta}$ : meia vida de eliminação plasmática; $\mathrm{T}_{1 / 2 \gamma}$ : meia vida de eliminação terminal; $\mathrm{K}_{10}$ : constante de eliminação; $\mathrm{K}_{12}$ e $\mathrm{K}_{13}$ : constantes de distribuição; $\mathrm{K}_{21}$ e $\mathrm{K}_{31}$ : constantes de redistribuição; A, B e C: interceptos; $\alpha, \beta$ e $\gamma$ : constante de eliminação compartimental. 
A maior $\mathrm{C}_{\max }$ no NANO está relacionada com o maior tempo para atingir a concentração plasmática máxima $\left(\mathrm{T}_{\max }\right)$, descrito para formulações com Solutol (KU \& VELAGALETI, 2010), parâmetro este não considerado em nosso estudo em razão do emprego de uma dose bolus, seguida de IC. O fato de a primeira colheita ter sido aos dois minutos após a administração do propofol resultou em $\mathrm{C}_{\max }$ distintos, pois a nanoemulsão necessita de maior tempo para liberação do propofol no plasma (TAMILVANAN, 2009).

Os valores de $\mathrm{C}_{\max }$ observados no NANO decorrem da menor afinidade do veículo pelos tecidos lipídicos, resultado confirmado pelo menor $\mathrm{V}_{\mathrm{dss}}$ em NANO. Essa formulação apresenta maior fração livre e menor distribuição tecidual (JUNG et al., 2010), resultado este diferente do observado por MOREY et al. (2006a) e LEE et al. (2009), que encontraram maior concentração plasmática na formulação em emulsão lipídica, resultado atribuído por LEE et al. (2009) ao maior volume de distribuição de sua microemulsão.

Não foram observadas diferença entre as ASC no EMU e NANO, assim como descrito por $\mathrm{CHO}$ et al. (2010). Entretanto, os valores no NANO foram maiores, refletindo as $\mathrm{Cp}$ encontradas em menor distribuição lipídica da nanoemulsão (JUNG et al., 2010). A maior ASC é resultado do veículo nanoemulsionado que propicia maior circulação do princípio ativo e distribuição tecidual específica (TORCHILIN, 2007). KIM et al. (2007) relatam alteração na distribuição da nanoemulsão, levando a maiores $\mathrm{Cp}$, fato confirmado no presente estudo.

O volume de distribuição central representa a capacidade do fármaco em se distribuir no plasma, sendo os valores de $3,75 \mathrm{~L} \mathrm{~kg}^{-1}$ para EMU e de $2,38 \mathrm{~L}$ $\mathrm{kg}^{-1}$ para NANO maiores que os $1,4 \mathrm{~L} \mathrm{~kg}^{-1}$ encontrados por ZORAN et al. (1993) e 1,0 $\mathrm{L} \mathrm{kg}^{-1} \mathrm{de}$ COCKSHOTT et al. (1992) para a emulsão lipídica, sendo que LEE et al. (2009) encontraram valores de $\mathrm{V}_{1}$ de 4,4L para uma microemulsão e de $3,6 \mathrm{~L}$ para emulsão lipídica, que resultam em menores volumes que os do presente estudo. Segundo BETHS et al. (2001), as diferenças em relação ao $\mathrm{V}_{1}$ estão relacionadas com as metodologias de cada estudo. Os volumes de distribuição do segundo e terceiro compartimento são menores que os encontrados por LEE et al. (2009), resultado este atribuído às características do veículo da nanoemulsão.

Os volumes de distribuição no estado de equilíbrio $\left(\mathrm{V}_{\mathrm{dss}}\right)$ de $44,66 \mathrm{e} 21,4 \mathrm{~L} \mathrm{~kg}^{-1}$,respectivamente, para EMU e NANO, refletem diretamente o caráter lipofílico do veículo da emulsão lipídica que apresenta maior distribuição tecidual quando comparado à nanoemulsão, assim como relatado por KIM et al. (2007).

$\mathrm{O}$ menor $\mathrm{V}_{\mathrm{dss}}$ reflete a maior concentração plasmática da nanoemulsão e maior efeito clínico, característica confirmada por CORRÊA (2010), que encontrou valores menores de débito cardíaco, índice cardíaco, volume sistólico, índice sistólico, índice do trabalho ventricular esquerdo em cães anestesiados com a mesma nanoemulsão deste estudo.

Os valores de clearance metabólico de $81 \pm 28 \mathrm{~mL} \mathrm{~kg}^{-1} \mathrm{~min}^{-1}$ para EMU e de $48 \pm 29 \mathrm{~mL} \mathrm{~kg}^{-1}$ min $^{-1}$ para NANO são semelhantes aos descritos por NOLAN et al. (1993) e LEE et al. (2009), sugerindo semelhanças no metabolismo das formulações. Entretanto, o grande desvio padrão impossibilita uma análise mais detalhada desse parâmetro farmacocinético.

Os valores de constante de eliminação $\mathrm{k}_{10}$ foram 0,0253min para EMU e 0,0207min para NANO, resultados menores que os encontrados por LEE et al. (2009) de $0,135 \mathrm{~min}$ e por COCKSHOTT et al. (1992) de 0,034min. Essa diferença é justificada pela taxa de eliminação $\gamma$ de $0,08 \mathrm{~h}^{-1}$ (Tabela 1), enquanto LEE et al. (2009) encontraram uma taxa de eliminação de $0,36 \mathrm{~h}^{-1}$.

Não foi observada diferença estatística entre os tempos de meia-vida de distribuição $\left(\mathrm{t}_{1 / 2 \alpha}\right)$ de EMU e NANO com tempos de 2,06 $\pm 1,34$ e $4,03 \pm 2,27 \mathrm{~min}$, respectivamente. Esses valores podem ser atribuídos aos valores de $\mathrm{C}_{\max }$ encontrados onde a maior $\mathrm{Cp}$ em NANO necessita de um maior tempo para ser distribuída (RAVENELLE et al., 2008). Os valores obtidos de $t_{1 / 2 \alpha}$ são semelhantes aos relatados por LEE et al. (2009) de 3,05min para uma microemulsão e 3,16min para a emulsão lipídica, sendo que CHOCKSHOTT et al. (1992) encontraram valores de $t_{1 / 2 \alpha}$ variando de 4 a 7 minutos e NOLAN et al. (1993), tempos de $t_{1 / 2 \alpha}$ de 1 a 2 minutos para a emulsão lipídica.

Os valores de meia vida de eliminação plasmática $\left(\mathrm{t}_{1 / 2 \beta}\right)$ de 58 e 52 minutos, respectivamente, para EMU e NANO foram maiores que os 32 minutos descritos por LEE et al. (2009) para uma microemulsão e menores que os descritos por HALL et al. (1997) de 131 minutos. A meia-vida de eliminação terminal $\left(\mathrm{t}_{1 / 2 \gamma}\right)$ foi maior que a calculada por LEE et al. (2009). Segundo HALL et al. (1997), as diferenças nos valores de $t_{1 / 2 \beta}$ e $t_{1 / 2 \gamma}$ são corriqueiras, pois se trata de um fármaco altamente lipossolúvel e com elevado clearance que apresenta metabolismo hepático, portanto, alterações de fluxo sanguíneo hepático e de débito cardíaco influenciam nos tempos de meia-vida $\beta$ e $\gamma$. 


\section{CONCLUSÃO}

Com base nos resultados obtidos e na metodologia proposta, é possível concluir que o propofol em nanoemulsão não apresenta diferenças farmacocinéticas em comparação à formulação tradicional em emulsão lipídica. Os parâmetros farmacocinéticos calculados permitem empregar o propofol em nanoemulsão no regime de infusão contínua.

\section{AGRADECIMENTOS}

À Coordenação de Aperfeiçoamento de Pessoal de Nível Superior (CAPES), pela concessão de bolsa de estudo; à Ourofino Saúde Animal, pelo apoio financeiro; e ao laboratório de Farmacocinética da Universidade Federal de Santa Catarina (UFSC).

\section{COMITÊ DE ÉTICA}

Este estudo foi aprovado pelo Comitê de Ética e Bem Estar Animal (CETEA) da instituição de origem, sob o protocolo $1.32 / 10$.

\section{REFERÊNCIAS}

BETHS, T. et al. Evaluation and optimization of target- controlled infusion system for administering propofol to dogs as part of a total intravenous anaesthesia technique during dental surgery. Veterinary Record, n.148, p.198-203, 2001. Disponível em: <http://veterinaryrecord.bmj.com/content/148/7/198>. Acesso em: 23 abr. 2012. doi: 10.1136/vr.148.7.198.

CHO, J. et al. Formulation and Evaluation of an Alternative Triglyceride-free Propofol Microemulsion. Archives of Pharmacal Research, v.33, n.9, p.1375-1387, 2010. Disponível em: <http://www.springerlink.com/content/y342121251902824/>. Acesso em: 23 abr. 2012. doi: 10.1007/s12272-010-0911-0.

CLEALE, R.M. et al. Pharmacokinetic and pharmacodynamic evaluation of propofol administered to cats in a novel, aqueous, nano-droplet formulation or as an oil-in-water macroemulsion. Journal of Veterinary Pharmacology and Therapeutics, v.32, p. 436-445, 2009. Disponível em: <http://onlinelibrary.wiley.com/ doi/10.1111/j.1365-2885.2009.01059.x/full>. Acesso em: 23 abr. 2012. doi: 10.1111/j.1365-2885.2009.01059.x.

COCKSHOTT, I.D. et al. The pharmacokinetics of propofol in laboratory animals. Xenobiotica, v.22, n.3, p.269-275, 1992.

CORRÊA, A.L. Avaliação hemodinâmica e metabólica de uma nanoemulsão de propofol em cães. 2010. 150f. Dissertação (Mestrado em Ciência Animal) - Curso de Pós-graduação em Ciência Animal, Centro de Ciências Agroveterinárias, Universidade do Estado de Santa Catarina, SC.

DATE, A.A; NAGARSENKER, M.S. Parenteral microemulsions: an overview. International Journal of Pharmaceutics, v.355, n.1-2, p.19-30, 2008. Disponível em: <http://www.ncbi.nlm.nih. gov/pubmed/18295991>. Acesso em: 23 abr. 2012. doi: 10.1016/j. ijpharm.2008.01.004.
GEHRCKE, M.I. Determinação das doses letal 50 e 100 do propofol em nanoemulsão ou em emulsão lipídica pela via intraperitoneal em camundongos. In: ENCONTRO DE ANESTESIOLOGIA VETERINÁRIA, 10., 2011, Campos do Jordão, SP. Anais... Campos do Jordão: Ourotour, 2011. p.14.

HALL, L.W. et al. Disposition of propofol after medetomidine premedication in beagle dogs. Journal of Veterinary Anaesthesia, v.24, n.1, p.23-29, 1997. Disponível em: <http://onlinelibrary. wiley.com/doi/10.1111/j.1467-2995.1997.tb00264.x/pdf>. Acesso em: 23 abr. 2012. doi: 10.1111/j.1467-2995.1997.tb00264.x.

JUNG, J.A. et al. Effectiveness, safety, and pharmacokinetic and pharmacodynamic characteristics of microemulsion propofol in patients undergoing elective surgery under total intravenous anaesthesia. British Journal of Anaesthesia, v.104, n.5, p.563576, 2010. Disponível em: <http://bja.oxfordjournals.org/ content/104/5/563.full.pdf+html>. Acesso em: 23 abr. 2012. doi: $10.1093 / \mathrm{bja} / \mathrm{aeq} 040$.

KIM, K.M. et al. Pharmacokinetics and pharmacodynamics of propofol microemulsion and lipid emulsion after an intravenous bolus and variable rate infusion. Anesthesiology, v.106, p.924-934, 2007. Disponível em: <http://journals. lww.com/anesthesiology/pages/results.aspx ?k=10.1097/01. anes.0000265151.78943. af \&Scope $=$ AllIssues \& txtKeywor $\mathrm{ds}=10.1097 / 01$.anes.0000265151.78943.af $>$. Acesso em: 23 abr. 2012. doi: 10.1097/01.anes.0000265151.78943.af.

KOTANI, Y. et al. The experimental and clinical pharmacology of propofol, an anesthetic agent with neuroprotective properties. CNS Neuroscience \& Therapeutics, v.14, n.2, p.95-106, 2008. Disponível em: <http://onlinelibrary.wiley.com/doi/10.1111/ j.1527-3458.2008.00043.x/pdf>. Acesso em: 23 abr. 2012. doi: 10.1111/j.1527-3458.2008.00043.x.

KU, S.; VELAGALETI, R. Solutol HS15 as a novel excipient. Pharmaceutical Technology, p.108-110, 2010. Acesso em: 23 abr. 2012. Online. Disponível em: $<$ http://pharmtech.findpharma. com/pharmtech/Ingredients/Solutol-HS15-as-a-Novel-Excipient/ ArticleStandard/Article/detail/694692>.

LEE, S.H. et al. Pharmacokinetics and pharmacodynamics of a new reformulated microemulsion and the long-chain triglyceride emulsion of propofol in beagle dogs. British Journal of Pharmacology, v.158, n.8, p.1982-1995, 2009. Disponível em: <http://www.ncbi.nlm.nih.gov/pmc/articles/PMC2807660/pdf/ bph0158-1982.pdf>. Acesso em: 23 abr. 2012. doi: 10.1111/j.14765381.2009.00509.x.

MOREY, T.E. et al. Anesthetic properties of a propofol microemulsion in dogs. Anesthesia and Analgesia, v.103, n.4, p.882-887, 2006a. Disponível em: <http://www.pubmedcentral. nih.gov/articlerender.fcgi artid=2999695\&tool=pmcentrez\&re ndertype=abstract $>$. Acesso em: 23 abr. 2012. doi: 10.1213/01. ane.0000237126.57445.80.

MOREY, T.E. et al. Preparation and anesthetic properties of propofol microemulsions in rats. Anesthesiology, v.104, n.6, p.1184-1190, 2006b. Disponível em: <http://journals.lww.com/ anesthesiology/Fulltext/2006/06000/Preparation_and_Anesthetic_ Properties_of_Propofol.13.aspx>. Acesso em: 23 abr. 2012. doi: 10.1097/01.anes.0000265151.78943.af.

NOLAN, A. et al. The effects of halothane and nitrous oxide on the pharmacokinetics of propofol in dogs. Journal of 
Veterinary Pharmacology \& Therapeutics, v.16, p.335342, 1993. Disponível em: <http://onlinelibrary.wiley.com/ doi/10.1111/j.1365-2885.1993.tb00180.x/pdf>. Acesso em: 23 abr. 2012. doi: 10.1111/j.1365-2885.1993.tb00180.x.

RAVENELLE, F. et al. Anaesthetic effects of propofol polymeric micelle: a novel water soluble propofol formulation. British Journal of Anaesthesia, v.101, n.2, p.186-193, 2008. Disponível em: <http://bja.oxfordjournals.org/content/101/2/186.full>. Acesso em: 23 abr. 2012. doi: 10.1093/bja/aen147.

TAMILVANAN, S. Formulation of multifunctional oil-in-water nanosized emulsions for active and passive targeting of drugs to otherwise inaccessible internal organs of the human body. International Journal of Pharmaceutics, v.381, n.1, p.62-76, 2009. Disponível em: <http://www.sciencedirect.com/science/ article/pii/S0378517309005110>. Acesso em: 23 abr. 2012. doi: 10.1016/j.ijpharm.2009.08.001
TORCHILIN, V.P. Nanocarriers. Pharmaceutical Research, v.24, p.2333-2334, 2007. Disponível em: <http://www.springerlink com/content/07uv3443qm650x51/fulltext.pdf>. Acesso em: 23 abr. 2012. doi: 10.1007/s11095-007-9463-5.

TRAPANI, A. et al. Evaluation of new propofol aqueous solutions for intravenous anesthesia. International Journal of Pharmaceutics, v.278, n.1, p.91-8, 2004. Disponível em: <http:// www.sciencedirect.com/science/article/pii/S0378517304001528>. Acesso em: 23 abr. 2012. doi: 10.1016/j.ijpharm.2004.02.028.

WHITE, P.F. Propofol: its role in changing the practice of anesthesia. Anesthesiology, v.109, n.6, p.1132-1136, 2008. Disponível em: <http://journals.1ww.com/anesthesiology/Fulltext/2008/12000/ Propofol__Its_Role_in_Changing_the_Practice_of.27.asp $\mathrm{x}>$. Acesso em: 23 abr. 2012. doi: 10.1097/ALN.0b013e31818ddba8.

ZORAN, D.L. et al. Pharmacokinetics of propofol in mixed breed dogs and Greyhounds. American Journal of Veterinary Research, v.54, n.5, p.755-760, 1993. 